\title{
Secondary Precipitation within the Cementite Phase of Reactor Pressure Vessel Steels
}

\author{
J. Zelenty ${ }^{1}$, G.D.W. Smith ${ }^{1}$, K. Wilford ${ }^{2}$, J.M. Hyde ${ }^{3}$, M.P. Moody ${ }^{1}$ \\ ${ }^{1}$ University of Oxford, Department of Materials, Oxford, United Kingdom OX1 3PH \\ ${ }^{2}$ Rolls Royce, P.O. Box 2000, Derby, United Kingdom DE21 7XX \\ ${ }^{3}$ National Nuclear Laboratory, Harwell, B168 Didcot, Oxon, United Kingdom OX11 0QA
}

\begin{abstract}
Solute clustering is known to play a significant role in the embrittlement of reactor pressure vessel (RPV) steels. In this study, previously unobserved manganese-, nickel-, and silicon-rich precipitates in the cementite phase of a thermally aged, low copper RPV steel are characterized at the atomic scale via atom probe tomography. Additionally, copper-rich precipitates are observed in the cementite phase of a thermally aged, high copper RPV steel. Due to their secluded nature, these secondary precipitates could be utilised as indicators of the timetemperature exposure of RPVs and assist in assessing remnant lifetime.
\end{abstract}

Keywords: RPV steels, cementite, precipitation, atom probe tomography, thermal ageing

In a light water reactor, the reactor pressure vessel (RPV) must withstand high temperatures, high pressures, and long-term exposure to high-energy neutrons. As they are prohibitively expensive to replace, RPVs are typically the limiting factor in reactor lifetime.

In order to improve the initial properties of RPV steels, solute is added to the iron. For example, Ni strengthens the steel at low temperatures; Si de-oxidises the steel; and Mn, via the formation of $\mathrm{MnS}$, attenuates brittleness [1]. While these solutes are critically important to the structural integrity of the RPV, solute clustering is known to play a significant role in its embrittlement [2]. Copper-rich precipitates (CRPs) form in steels with greater than 0.1 at.\% copper at typical RPV operating temperatures (on the order of $300^{\circ} \mathrm{C}$ ) [3]. Significant previous research has shown that CRPs are a key cause of hardening and the eventual degradation of RPVs [3-6]. Due to the formation of CRPs, new generation RPVs have been engineered to contain less than 0.1 at.\% copper [4]. Although these steels are no longer subject to the detrimental effects of CRPs, attention has now turned to the potential hardening and embrittlement effects of manganese-, nickel-, and silicon-rich precipitates (MNPs). Therefore, it remains imperative to track how solute is distributed within the microstructure throughout the lifetime of the RPV.

Although CRPs within the matrix of RPV steels have been extensively studied [4-9], there is relatively little in the published literature regarding CRPs within carbides. Copper-rich solute clusters located within carbides were first reported by Khalid and Edmonds utilising TEM $[10,11]$. However, the material analysed in that study was a high-alloy hypereutectoid steel containing large amounts of Mn and thus significantly different from the model RPV steels investigated in this study. Through the use of scanning transmission electron microscopyenergy-dispersive spectrometry (STEM-EDS) they showed that these precipitates were high in copper. However, an extensive chemical characterisation was not conducted. MNPs located within the matrix of low copper steels have also been extensively studied [12-16], and although MNPs have been observed at heterogeneous nucleation sites after thermal ageing [4], previous work has not revealed any secondary MNPs located within carbides in the new generation RPV steels. 
In this study, CRPs and MNPs were observed within the cementite phase of a high copper and low copper RPV steel, respectively, following long term thermal ageing. Atom probe tomography (APT) was used to characterise the precipitates and obtain more detailed chemical information. This was made possible through APT's unique combination of atomic sensitivity and sub-nanometer 3D spatial resolution [17].

The high and low copper RPV type steels analysed in this study were provided by Rolls-Royce Plc. (Table 1). After rolling, the steels were subjected to an initial heat treatment as $25 \mathrm{~mm}$ thick plates. The plates underwent austenitisation at approximately $920^{\circ} \mathrm{C}$ for 1 hour followed by an air cool. Carbides typically form during this step in the heat treatment [18]. The plates were then tempered at approximately $600^{\circ} \mathrm{C}$ for 5 hours followed by a final air cool.

Following this forging and processing, the steels were thermally aged for 11,700 hours at $405^{\circ} \mathrm{C}$ (high copper) or $365^{\circ} \mathrm{C}$ (low copper). Although RPV's typically operate at $290^{\circ} \mathrm{C}$, higher temperatures are utilised in this study to expedite cluster formation. The steels were kept in vacuum during the thermal ageing treatment to avoid oxidation. Upon removal from the furnaces, the steels were quenched in water.

Specimens for TEM were prepared from $3 \mathrm{~mm}$ disks, which were ground down to a thickness of approximately $90 \mu \mathrm{m}$. The specimens were then electropolished using $90 \%$ perchloric acid with a TenuPol Electropolisher. The TEM was conducted on a Jeol 2100.

Specimens analysed using APT were prepared either by electropolishing or site-specific focused ion beam (FIB) lift-out. The former utilised $0.5 \mathrm{~mm}$ by $0.5 \mathrm{~mm}$ matchsticks, which were first-stage electropolished using $25 \%$ perchloric acid, then further sharpened (second-stage electropolished) using 2\% perchloric acid in butoxyethanol [19]. The latter was carried out on a Zeiss Auriga FIB-SEM. The site-specific FIB lift-out technique is described in detail by Thompson et al. [20]. Atom probe experiments were conducted using voltage mode with a pulse fraction of $20 \%$ on a LEAP $3000 \mathrm{X}$ HR. The $3 \mathrm{D}$ reconstruction of the data was created using IVAS $^{\mathrm{TM}}$ 3.6.6, a software based on a standard reverse-projection algorithm [21].

APT analysis is complicated by the fact that the mass spectra of RPV steels can contain several peak overlaps, the most notable of which is the ${ }^{58} \mathrm{Ni}$ and ${ }^{58} \mathrm{Fe}$ peak overlap. Typically, the relative contributions to these peaks are deconvolved using known natural isotopic abundances. Generally, this calculation is applied to the entire dataset. However, in this study the peak deconvolution depended heavily on local chemistry and was therefore carried out separately for the precipitates, carbide matrix, and iron matrix.

Another potential peak overlap is $\mathrm{Si}^{28}$ and $\mathrm{N}^{14}$. However, the nitrogen content in these model RPV steels is very low. Furthermore, the limited amount of nitrogen that is present in these steels is largely tied up in aluminum nitride particles and molybdenum carbo-nitrides. Regardless, it is possible to calculate the expected Si contributions to the relevant overlapping peaks (14 Da and $15 \mathrm{Da}$ ) using isotope abundancies. From these calculations it was determined that the nitrogen content was negligible in the three atom probe samples presented.

In this study, CRPs were observed within the cementite phase of thermally aged high copper RPV steels. In order to confirm that these particles were located within the cementite, rather than above or below, a series of under-, in-, and, over-focus TEM images were obtained. Since the precipitates came in and out of focus with the carbide, it was determined that they were 
in fact located within this phase. One of the in-focus TEM images is shown in Figure 1. By visual inspection the precipitates appear to be randomly distributed throughout the cementite as well as the iron matrix. At the current ageing time, it is believed that the precipitates are clusters of solute within the cementite lattice and that they lack a crystal structure of their own. However, electron diffraction was not possible on the Jeol 2100 available at Oxford due to the small size of the precipitates. Therefore, this hypothesis could not be verified at this time.

TEM analyses of the as-received high and low copper steels showed no evidence of precipitates within the cementite phase [22]. This suggests that these features form during thermal ageing. Additionally, previous work determined that $\mathrm{M}_{3} \mathrm{C}$ (the metallic constituent of this phase being predominantly $\mathrm{Fe}$ ) was the most common carbide in both the high and low copper steels [22]. These results are consistent with the APT data obtained in this study as all three of the carbides observed were cementite $\left(\mathrm{Fe}_{3} \mathrm{C}\right)$.

APT was utilised to obtain a more detailed chemical characterisation of the CRPs found in the thermally aged samples. In agreement with the TEM results, APT analyses showed CRPs within the cementite phase of the thermally aged high copper RPV steel (Figure 1). These precipitates were approximately $5 \mathrm{~nm}$ in diameter and found in manganese-rich cementite.

The average composition of the CRPs, the matrix, and the carbide are shown in Table 2. The secondary precipitates contain elevated levels of copper, nickel, and silicon. This is illustrated by a 'proxigram' analysis, which plots the chemical distribution as a function of distance from the precipitate/carbide interface, in Figure 1. Since trajectory aberrations can significantly influence matrix element levels within precipitates, the average composition for CRPs without the cementite matrix elements, Fe and $\mathrm{C}$, is also provided.

In the thermally aged low copper RPV steel, MNPs were observed in two APT analyses, one of which is shown in Figure 2. These precipitates were approximately $5 \mathrm{~nm}$ in diameter and found in Mo- and Mn-rich cementite phases. A single MNP is highlighted in Figure 2 in order to measure its composition using a 1D concentration profile. The average composition of the precipitates, as well as the matrix and carbide compositions, are shown in Table 3 . The compositions are a weighted average of the two atom probe analyses of the low copper steel. Forty precipitates were analyzed in total; eight and thirty-two precipitates in each APT reconstruction, respectively.

The MNP solute compositions from the two analyses were consistent except for $\mathrm{Si}$, which varied significantly between the two datasets (16.5 at.\% and 8.2 at.\%). Similarly, it was observed that the Si content within the cementite phase of the two samples was also different. This variation in $\mathrm{Si}$ content is likely due to the fact that cementite plates form at different times in the cooling process and therefore at different temperatures. At lower temperatures more $\mathrm{Si}$ becomes trapped within the quickly forming cementite phase, while at higher temperatures more $\mathrm{Si}$ is able to diffuse into the matrix. The Si content of the MNPs was strongly correlated to the $\mathrm{Si}$ levels in the cementite phases in which they were located: higher Si content within the cementite phase led to MNPs higher in Si and vice versa.

Additionally, the MNPs observed contained relatively small amounts of Mn. Since Mn is a strong carbide former, it is hypothesised that the $\mathrm{Fe}_{3} \mathrm{C}$ matrix extracts much of the $\mathrm{Mn}$ out of the MNPs. Ni and Si, however, are weak carbide formers and thus are the predominant species found within the precipitates. 
Cementite forms rapidly at large supersaturations in pearlitic and bainitic steels [23]. It is therefore proposed that during cooling after austenitisation, when carbides typically form, solute becomes trapped within the quickly forming phase. Without time for the redistribution of these solutes during cooling they become trapped in the cementite. Driving forces for precipitation come into play during thermal ageing when the kinetics of the system are increased considerably. Solutes, such as $\mathrm{Ni}, \mathrm{Cu}$, and $\mathrm{Si}$, which have a low solubility in cementite, readily precipitate out of solution. Conversely, elements which have a high solubility, such as Mn and Mo, remain in solid solution within the cementite. This hypothesis explains the observed chemical compositions as well as the absence of precipitates in the as-received material prior to thermal ageing.

The total amount of solute trapped within the secondary precipitates was estimated. An upper bound of the carbide volume fraction can be obtained by assuming that all of the carbon is consumed to form cementite and all atoms occupy the same volume regardless of chemical identity. Using these approximations, upper bounds for the carbide volume fraction were 3.6 at. $\%$ and 4.8 at. $\%$ in the high and low copper steels, respectively.

This estimate can be refined for the low copper steel by comparing the nominal, matrix, and cementite compositions for each element. Specifically, the difference between matrix and nominal compositions has been plotted against the difference between matrix and cementite compositions in Figure 3. The resulting points were then fitted with a line, the slope of which was used to estimate the volume fraction of cementite, giving approximately 4.5 at. $\%$ for the low copper steel. Due to insufficient data, a more precise estimate of the volume fraction for the high copper steel was not obtained.

From these volume fractions (3.6 at.\% and 4.5 at.\%) and the chemical compositions obtained from APT it was possible to estimate the amount of solute trapped within cementite for both the high and low copper steels (Table 4). It should be noted that any significant effects on the microstructure will already have been implicitly accounted for in estimates of RPV lifetime as all RPV steels contain carbides and thus presumably these secondary precipitates.

The diffusion distances of the various solutes within iron were calculated and range from $1.3 \times 10^{-8} \mathrm{~m}^{2} / \mathrm{s}$ to $6.4 \times 10^{-5} \mathrm{~m}^{2} / \mathrm{s}$ at $405^{\circ} \mathrm{C}$. These values can be found in Table 5. The activation energies and the diffusion coefficients of solutes in cementite were not readily available, however solute diffusion distances in cementite are expected to be approximately an order of magnitude smaller than in iron.

Due to the secluded nature of secondary precipitates, CRPs and MNPs within cementite could potentially be used as an indicator for the time-temperature exposure of the steel. Since these precipitates are located within the cementite phase, they are isolated from interactions with dislocations which could alter their morphology. Additionally, due to the relatively small diffusion distances of solute within cementite, the cementite phase is a comparatively isolated system from the rest of the steel. Monitoring the growth and coarsening of these secondary precipitates could be useful in tracking the time-temperature exposure of RPVs. This potential application is worth exploring as it could prove to be an extremely useful tool in assessing remnant lifetime.

In summary, CRPs and MNPs were observed within the cementite phase of high and low copper RPV steels, respectively, using APT. Although CRPs have been previously observed in 
cementite within high-alloy steels, this is the first study in which MNPs have been observed in cementite [10]. This research has shown that a range of precipitation can take place within the cementite phase of thermally aged RPV steels. Additionally, there is potential for utilising these secondary precipitates as indicators of the time-temperature exposure of RPVs, thus assisting in assessing remnant lifetime.

\section{Bibliography}

[1] B.L. Bramfitt, A.O. Benscoter, Metallographer's Guide: Irons and Steels. Materials Park, OH: ASM International, 2001.

[2] K.C. Russell, L.M. Brown, Acta Metallurgica. 20 (1972) 969-74.

[3] G. Salje, M. Feller-Kniepmeier, Journal of Applied Physics. 48 (1977) 1833.

[4] P.D. Styman, Atomic Scale Studies of Thermally Aged Reactor Pressure Vessel Steels, D.Phil. Thesis. University of Oxford, 2012.

[5] G.R. Odette, G.E. Lucas, Radiation Effects and Defects in Solids: Incorporating Plasma Science and Plasma Technology. 144 (1998) 189-231.

[6] E.D. Eason, G.R. Odette, R.K. Nanstad, T. Yamamoto, “A Physically Based Correlation of Irradiation-Induced Transition Temperature Shifts for RPV Steels," ORNL/TM-2006/530, 2006.

[7] M.K. Miller, P. Pareige, M.G. Burke, Materials Characterization. 44 (2000) 235-254.

[8] A. Deschamps, M. Militzer, W.J. Poole, ISIJ International. 41 (2001) 196-205.

[9] P.J. Othen, M.L. Jenkins, G.D.W. Smith, Proceedings of the International Conference on Martensitic Transformations. Monterey, California, USA. 1993.

[10] F.A. Khalid, D.V. Edmonds, Metallurgical Transactions A. 24A (1993) 781.

[11] F.A. Khalid, D.V. Edmonds, Journal of Materials Processing Technology. 72 (1997) 434436.

[12] P. Pareige, J.C. Van Duysen, P. Auger, Applied Surface Science. 67 (1993) 342-374.

[13] P. Auger, P. Pareige, M. Akamatsu, J.C. Van Duysen, Journal of Nuclear Materials. 211 (1994) 194-201.

[14] C.L. Liu, G.R. Odette, B.D. Wirth, G.E. Lucas, Materials Science and Engineering. A238 (1997) 202-209.

[15] P.D. Styman, J.M. Hyde, K. Wilford, A. Morley, G.D.W. Smith, Progress in Nuclear Energy. 57 (2012) 86-92.

[16] A. Wagner, A. Ulbricht, F. Bergner, E. Altstadt, Nuclear Instruments and Methods in Physics Research B. 280 (2012) 98-102.

[17] B. Gault, M.P. Moody, F. De Geuser, A. La Fontaine, L.T. Stephenson, D. Haley, S.P. Ringer, Microscopy and Microanalysis. 16 (2010) 99-110. 
[18] G. Roberts, G. Krauss, R. Kennedy, Tool Steels. Metals Park, OH: American Society for Metals, 1998.

[19] M.K. Miller, G.D.W. Smith, A. Cerezo, M.G. Hetherington, Atom Probe Field Ion Microscopy Monographs on the Physics and Chemistry of Materials. Oxford: Oxford University Press, 1996.

[20] K. Thompson, D. Lawrence, D.J. Larson, J.D. Olson, T.F. Kelly, B. Gorman, Ultramicroscopy. 107 (2007) 131-139.

[21] B. Gault, D. Haley, F. de Geuser, M.P. Moody, E.A. Marquis, D.J. Larson, B.P. Geiserm, Ultramicroscopy. 111 (2011) 448-457.

[22] J.M. Titchmarsh, "The microstructural characterisation of six experimental ferritic steel alloys," Rolls Royce Report OM 11/598, 2012.

[23] H.K.D.H. Bhadeshia, R. Honeycombe, Steels: Microstructure and Properties. London: Butterworth-Heinemann, 2006.

[24] W.F. Gale, T.C. Totemeier, Smithells Metals Reference Book. 8th ed. London: ButterworthHeinemann, 2004. 